\title{
A Study for Improving the Positioning Accuracy of DGPS Based on Multi-Reference Stations by Applying Exponential Modeling on Pseudorange Corrections
}

\author{
Koon-Tack Kim ${ }^{1 \dagger}$, Kwan-Dong Park², Eunsung Lee', Moon Beom Heo ${ }^{1}$ \\ ${ }^{1}$ Satellite Navigation Team, Korea Aerospace Research Institute, Daejeon 305-806, Korea \\ ${ }^{2}$ Department of Geoinformatic Engineering, Inha University, Incheon 402-751, Korea
}

\begin{abstract}
In this paper, a pseudorange correction regeneration algorithm was developed to improve the positioning accuracy of DGPS using multi-reference stations, and the optimal minimum number of reference sites was determined by trying out different numbers of reference. This research was conducted using from two to five sites, and positioning errors of less than $1 \mathrm{~m}$ were obtained when pseudorange corrections are collected from at least four reference stations and interpolated as the pseudorange correction at the rover. After determining the optimal minimum number of reference stations, the pseudorange correction regeneration algorithm developed was tested by comparison with the performance of other algorithms. Our approach was developed based on an exponential model. If pseudorange corrections are regenerated using an exponential model, the effect of a small difference in the baseline distance can be enlarged. Therefore, weights can be applied sensitively even when the baseline distance differs by a small amount. Also weights on the baseline distance were applied differently by assigning weights depending on the difference of the longest and shortest baselines. Through this method, the positioning accuracy improved by $19 \%$ compared to the result of previous studies.
\end{abstract}

Keywords: DGPS, pseudo-range, exponential model, interpolation

\section{INTRODUCTION}

The Differential Global Positioning System (DGPS) was first developed before 2000 in the private sector to minimize the effect of Selective Availability (SA) which generates intentional noise on purpose for limiting the use of GPS. The system could improve the accuracy of GPS, and various studies have been conducted using DGPS even after the withdrawal of SA. As for the studies related with DGPS, the DGPS was used for analyzing the approach and landing accuracy of aircraft (Koo et al. 2011), and a study was conducted which proposes a flight test method using DGPS (Lee et al. 2009). Also, the DGPS positioning result was used

Received Apr 01, 2013 Revised Apr 29, 2013 Accepted Apr 30, 2013

tCorresponding Author

E-mail: koontack@kari.re.kr

Tel: +82-42-870-3977 Fax: +82-42-860-2789 for evaluating the performance of risky driving decision device (Oh \& Lee 2010), and a study was conducted which uses DGPS for high-accuracy surveying as the availability evaluation of network DGPS positioning for various facilities management in dense housing area (Kim 2010). As the positioning accuracy of DGPS is higher than that of GPS, the DGPS is used in various fields such as aviation, surveying, transportation, and vessel navigation, and the demand is also gradually increasing.

The DGPS calculates an error by receiving a satellite signal at the reference station, and provides the error information to users, which enables the positioning accuracy of about $1 \mathrm{~m}$ level. Therefore, to use the DGPS, a reference station is necessary for calculating the error of GPS signal and providing the error information. In Korea, the DGNSS Central Office from the Ministry of Land, Transport, and Maritime Affairs provides the DGPS service by installing the DGPS reference station. The methods for 
providing error information to users include the Beacon method which uses a medium wave signal and the Network Transport of RTCM via Internet Protocol (NTRIP) method which uses the Internet. In recent years, as a transmission technique was developed which can provide DGPS error information using the FM DAta Radio Channel (DARC) and Digital Multimedia Broadcasting (DMB), many users are able to use the DGPS service.

The error information provided from the reference station includes the pseudorange correction (PRC). The pseudorange correction indicates the difference between the true range from the reference station whose coordinate is known accurately to each satellite and the pseudorange that is recorded in the observation data. Users can improve the positioning accuracy using the pseudorange correction provided from the reference station. As the pseudorange correction provided from the reference station has a high correlation depending on distance, the accuracy of pseudorange correction decreases when the distance between reference station and user increases. Therefore, in Korea, the pseudorange correction is provided by shortening the distance between reference station and user through installing multiple reference stations. However, this requires a great deal of manpower and cost for operating the reference station. When the pseudorange corrections provided from multiple reference stations are interpolated and regenerated as the pseudorange correction of user location, the accuracy of pseudorange correction depending on baseline distance can be improved, and through this, the national manpower and budget for installing and operating the reference station can be reduced. The examples of studies for regenerating as the pseudorange correction of user location using multiple reference stations, proposed in foreign countries, are the method that assigns statistical weight using Kalman filter (Tang et al. 1989), the Partial Derivative Algorithm method (Loomis et al. 1991), and the Minimum Variance Network DGPS Algorithm method (Mueller 1994). Also, there is a method which interpolates by assigning weight respectively on the baseline distance, satellite altitude angle, and error information generation time of reference stations (Abousalem 1997). In Korea, a study was conducted which improves the positioning accuracy by regenerating error information through assigning weight depending on the baseline distance between reference station and user (Bae et al. 2003). Also, the performance of DGPS based on multi-reference stations was analyzed by developing a linear interpolation algorithm (Song et al. 2006). However, the studies from foreign countries were performed prior to the withdrawal of SA and do not accord with the current environment without SA, and the interval between the reference stations is maximum $1680 \mathrm{~km}$ which is different from the domestic environment. The previous studies in Korea used only the Eocheong Island, Socheong Island, and Jumunjin reference stations and therefore have a disadvantage of bad geometry between the reference stations, and the accuracy is not high because the method which assigns weight simply depending on distance was used.

Hence, in this paper, the optimal number of reference stations, which accords with the domestic environment, is selected among the DGPS methods based on multireference stations using 2 5 reference stations, and the accuracy of DGPS positioning is improved by developing a pseudorange correction regeneration algorithm.

\section{A WEIGHTING METHOD DEPENDING ON BASELINE DISTANCE}

The DGPS based on multi-reference stations collects the pseudorange correction of reference stations at the same time, and regenerates the pseudorange correction of user location, which is then provided to users. While regenerating the pseudorange correction, the baseline of reference station and user location has a large effect on the pseudorange correction of user. As the GPS error factors have a high correlation depending on baseline distance, the pseudorange correction of user location should be regenerated by assigning more weight on the pseudorange correction of the reference station that is close to user and less weight on the pseudorange correction of the reference station that is far from user. However, when the weight is assigned by relatively comparing the difference depending on distance to regenerate the pseudorange correction in a small country such as the Korean Peninsula, the difference of baseline distance is not large, and therefore the weight depending on distance difference needs to be maximized using an exponential model. This is because a large difference can be obtained by maximizing a small difference in value using an exponent. The longest baseline distance of the Korean Peninsula is about $650 \mathrm{~km}$ from the Unification Observatory in Goseong-gun, Gangwon Province which is the northernmost point in the east to the Mara Island which is the southernmost point in the west. When the DGPS is used in the land of the Korean Peninsula, the position is obtained within the baseline distance of about $500 \mathrm{~km}$.

For the baseline distance of 50 450 km, Table 1 and Fig. 1 show the results when a simple inverse of baseline distance is used as the weight and when an inverse is used as the weight by applying an exponential model to baseline 
Table 1. 1/Baseline vs 1/exp (Baseline).

\begin{tabular}{cccccc}
\hline Baseline $(\mathbf{k m})$ & $\mathbf{5 0}$ & $\mathbf{1 5 0}$ & $\mathbf{2 5 0}$ & $\mathbf{3 5 0}$ & $\mathbf{4 5 0}$ \\
\hline $1 /$ Baseline & 0.0196 & 0.0066 & 0.0040 & 0.0025 & 0.0022 \\
\hline $1 / \exp ($ Baseline $)$ & $7.01 \times 10^{-23}$ & $2.64 \times 10^{-66}$ & $9.82 \times 10^{-110}$ & $3.65 \times 10^{-153}$ & $1.36 \times 10^{-196}$ \\
\hline
\end{tabular}
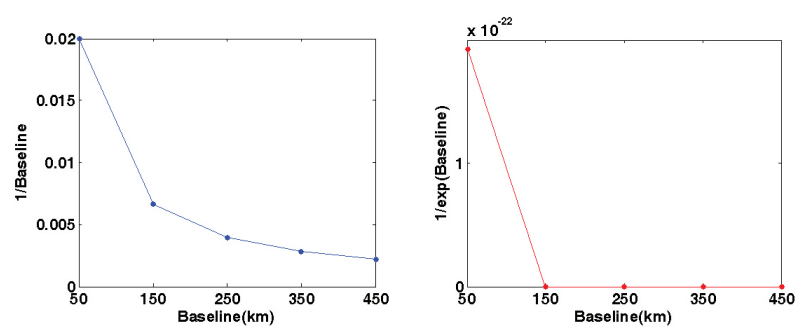

Fig. 1. 1/Baseline (left) vs 1/exp (Baseline) (right).

distance. Here, an inverse of baseline distance is used as the weight because the pseudorange correction should be regenerated by applying less weight to long baseline distance and more weight to short baseline distance. From Table 1, it is shown that the difference of $50 \mathrm{~km}$ baseline and $450 \mathrm{~km}$ baseline is about $10^{-1}$ for a simple inverse of baseline distance, and the difference is about $10^{-173}$ for an exponential model. Fig. 1 presents the numerical values of Table 1 , and it is found that the inverse of distance applying an exponential function shown in Fig. 1 (right) changes more abruptly than the inverse of distance shown in Fig. 1 (left). This indicates that the maximization of weight can be achieved using an exponential model when applying weight depending on baseline distance. Also, the correlation of GPS error factors depending on baseline distance can be maximized by assigning more weight on the reference station that is close to user and less weight on the reference station that is far from user.

\section{PSEUDORANGE CORRECTION REGENERATION ALGORITHM}

The pseudorange correction regeneration algorithm using an exponential model is based on the inverse distance weighting, and enables the maximization of weight using the algorithm developed in this paper rather than a simple inverse of distance in the process of assigning weight depending on the baseline distance difference of reference stations and user.

The pseudorange correction at user location can be calculated using Eq. (1), and $\delta \rho_{1}$ represents the pseudorange correction of 1-th satellite which is newly generated at user location and $\delta \rho_{1}^{j}$ represents the pseudorange correction of l-th satellite which is generated at $\mathrm{j}$-th reference station.
Table 2. Coefficient according to difference of longest and shortest baseline.

\begin{tabular}{ccccccc}
\hline LB - SB $(\mathrm{km})$ & $0 \sim 200$ & $200 \sim 250$ & $250 \sim 300$ & $300 \sim 350$ & $350 \sim 400$ & $400 \sim 500$ \\
\hline Coefficient $\left(\omega_{\mathrm{d}}\right)$ & 6 & 5 & 4 & 5 & 2 & 1
\end{tabular}

$\mathrm{k}$ represents the number of reference stations used for generating the pseudorange correction of user location. $\omega^{j}$ in Eq. (1) represents weight depending on baseline distance difference, and is shown in Eq. (2). In Eq. (2), B represents the baseline distance between $\mathrm{j}$-th reference station and user. LB represents the longest baseline having the longest distance among the baseline distances of reference stations and user location, and SB represents the shortest baseline. $\omega_{\mathrm{d}}$ represents a coefficient which is applied differently depending on the difference of the longest and shortest baselines, and is shown in Table 2. A large difference of the longest and shortest baselines indicates that the user is located close to a specific reference station. In this case, the weight value depending on baseline distance should be made large by applying a small coefficient $\left(\omega_{\mathrm{d}}\right)$. In contrast, when the baseline distances of the longest and shortest baselines are similar and the difference between the baseline distances is small, the weight value should be made small by applying a large coefficient $\left(\omega_{\mathrm{d}}\right)$.

$$
\begin{aligned}
& \delta \rho_{1}=\frac{\sum_{j=1}^{k} \delta \rho_{l}^{j} \omega^{j}}{\sum_{j=1}^{k} \omega^{j}} \\
& \omega^{j}=\exp \left(-\frac{B^{j} \times \omega_{d}}{L B-S B}\right)
\end{aligned}
$$

To test the coefficient depending on the difference of the longest and shortest baselines shown in Table 2, the pseudorange correction of rover station was regenerated by changing the coefficient of exponential model, and the result was analyzed by applying this to positioning. For the purpose of various analyses, the positioning result which uses the existing inverse distance weighting was also calculated and compared together. The Suwon (SUWN), Muju (MUJU), and Daegu (TEGN) stations were selected as the rover stations used for positioning among the continuously operating reference stations which are operated by the National Geographic Information Institute, and the notified coordinate provided by the National Geographic Information Institute was used as the true value for error calculation. The pseudorange correction of rover station was regenerated using the pseudorange corrections of Yeongdo (YNDO), Jeojin (JEOJ), Palmi Island (PALM), and Mara Island (MARA) reference stations of 
12:00 14:00, October 11, 2012. Table 3 shows the coefficient of exponential model depending on the longest baseline, the shortest baseline, and the difference of the longest and shortest baselines for each rover station, and Table 4 shows the positioning error depending on the coefficient of exponential model and the positioning error using the inverse distance weighting. The positioning error indicates the Root Mean Square (RMS) error of horizontal direction compared to the notified coordinate.

As shown in Table 3, the exponential model coefficient of 1 was used for the SUWN rover station as the baseline distance difference of the MARA reference station which is the longest baseline and the PALM reference station which is the shortest baseline is $417.8 \mathrm{~km}$, and the exponential model coefficient of 6 was used for the MUJU rover station as the difference of the longest and shortest baselines is $179.6 \mathrm{~km}$. For the TEGN rover station, the pseudorange correction was regenerated using the exponential model coefficient of 4 as the difference of the longest and shortest baselines is $290.2 \mathrm{~km}$. Regarding the results that applied the regenerated pseudorange correction to positioning, for the SUWN rover station, the highest positioning accuracy of $0.46 \mathrm{~m}$ was obtained when the exponential model coefficient of 1 was used, which reduces the error by $0.15 \mathrm{~m}$ compared to the inverse distance weighting. For the MUJU rover station, the highest positioning accuracy of $0.49 \mathrm{~m}$ was obtained when the exponential model coefficient of 6 was used, which reduces the error by $0.07 \mathrm{~m}$ compared to the inverse distance weighting. For the TEGN rover station, the highest positioning accuracy of $0.46 \mathrm{~m}$ was obtained when the exponential model coefficient of $3 \sim 6$ was used, which reduces the error by $0.30 \mathrm{~m}$ compared to

Table 3. Coefficient of each rover.

\begin{tabular}{ccccc}
\hline Rover & $\begin{array}{c}\text { LB } \\
\text { (Reference } \\
\text { station) }\end{array}$ & $\begin{array}{c}\text { SB } \\
\text { (Reference } \\
\text { station) }\end{array}$ & $\begin{array}{c}\text { LB-SB } \\
(\mathbf{k m})\end{array}$ & $\begin{array}{c}\text { Coefficient } \\
\left(\omega_{\mathrm{d}}\right)\end{array}$ \\
\hline SUWN & 466.8 (MARA) & 49.0 (PALM) & 417.8 & 1 \\
MUJU & 344.7 (MARA) & 165.1 (YNDO) & 179.6 & 6 \\
TEGN & 387.0 (MARA) & 96.8 (YNDO) & 290.2 & 4 \\
\hline
\end{tabular}

Table 4. Horizontal error according to coefficient (meter).

\begin{tabular}{cccc}
\hline Coefficient $\left(\omega_{\mathrm{d}}\right)$ & SUWN & MUJU & TEGN \\
\hline 1 & 0.46 & 0.55 & 0.64 \\
2 & 0.53 & 0.55 & 0.47 \\
3 & 0.64 & 0.54 & 0.46 \\
4 & 0.72 & 0.52 & 0.46 \\
5 & 0.77 & 0.50 & 0.46 \\
6 & 0.81 & 0.49 & 0.46 \\
\hline Inverse & & & \\
distance & 0.61 & 0.56 & 0.76 \\
weight & & & \\
\hline
\end{tabular}

the inverse distance weighting. In the case of TEGN rover station, it is thought that similar accuracy is observed for the coefficient of 3 or more because the difference of the longest and shortest baselines is $290.2 \mathrm{~km}$ which has the value close to $300 \mathrm{~km}$. Through the above results, it was possible to examine the validity of coefficient depending on the difference of the longest and shortest baselines shown in Table 2. In addition, the exponential model developed in this paper was tested by applying the coefficient in Table 2 to the accuracy test procedure suggested in chapter 5 .

\section{METHODS FOR SELECTING THE NUMBER OF REFERENCE STATIONS AND ANALYZING THE ALGORITHM ACCURACY}

In this paper, to select the optimal number of reference stations among the DGPS methods based on multireference stations, the positioning accuracy was evaluated using 2 5 reference stations. This paper used $2 \sim 5$ reference stations because when 6 or more reference stations are used, the highest accuracy can be achieved by regenerating the pseudorange correction utilizing the 2nd multiple linear regression analysis suggested by Kim \& Park (2011). To analyze the accuracy of the pseudorange correction regeneration algorithm developed in this paper, it was tested by comparing with the result of inverse distance weighting developed in the previous study.

\subsection{Selection of reference stations}

For selecting the reference stations which cover the entire Korean Peninsula, the reference stations located at the outermost region of the Korean Peninsula and the reference station located at the center of the Korean Peninsula were selected, and it was thought that the outer region can be covered by maximum 4 reference stations considering the topography of the Korean Peninsula. Also, as the tropospheric error changes from east to west and the ionosphere changes from west to east, the changes of troposphere and ionosphere could be quickly detected by selecting the reference stations located at the outermost region. The effect of orbit error which is sensitive to baseline distance was minimized by maintaining the longest baseline distance among the reference stations.

As shown in Fig. 2, the GPS error information was collected by selecting the YNDO, JEOJ, PALM, and MARA reference stations located at the outermost region and the Chungju (CCHJ) reference station located at the center of the Korean Peninsula among the reference stations operated 


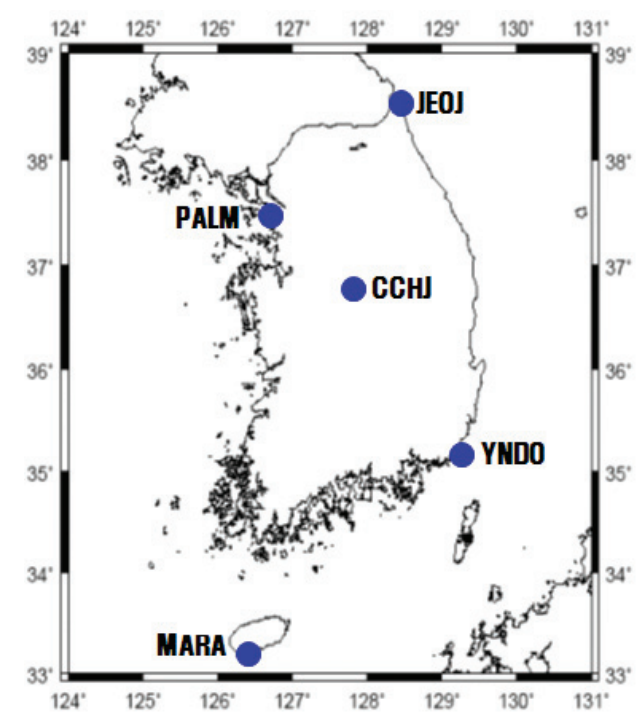

Fig. 2. YNDO, JEOJ, PALM, MARA, and CCHJ reference stations.

by the DGNSS Central Office.

In this paper, the pseudorange corrections of YNDO, JEOJ, PALM, MARA, and CCHJ reference stations of 03:00 05:00, October 11, 2012 based on UTC were collected. UTC is the Universal Time Coordinated and has a time difference of 9 hours compared to Korean Standard Time. UTC 03:00 05:00 corresponds to 12:00 14:00 in Korean Standard Time, and this is a time zone when the ionospheric error is the largest. This paper selected the time zone with the largest ionospheric error as the research subject time, and the accurate test of pseudorange correction regeneration algorithm was performed by utilizing the raw data without correcting the pseudorange correction of reference station via preprocessing procedure.

\subsection{Selection of rover stations}

To test the pseudorange correction regeneration algorithm proposed in this paper, the RINEX data was collected by selecting the MUJU continuously operating reference station located at the center of the Korean Peninsula as the rover station among the reference stations operated by the National Geographic Information Institute. The collected RINEX data has an interval of 30 seconds, and total 240 epochs were used for the research subject time, from 03:00 to 05:00.

\section{ANALYSIS RESULT}

In this paper, to improve the positioning accuracy of
DGPS using a minimum number of multi-reference stations, the number of reference stations, which shows the highest accuracy, was selected by changing the number of reference stations, and the positioning accuracy was evaluated by selecting the MUJU continuously operating reference station as the rover station. Also, it was tested by comparing with the result of inverse distance weighting developed in the previous study and the positioning result of DGPS based on single reference station.

\subsection{Comparison of positioning accuracy depending on the number of reference stations}

To compare the positioning accuracy depending on the number of reference stations, the pseudorange correction of rover station was regenerated using 2 5 reference stations. The MUJU continuously operating reference station located at the center of the Korean Peninsula was selected as the rover station among the reference stations operated by the National Geographic Information Institute. The YNDO, JEOJ, PALM, MARA, and CCHJ reference stations were used as the 5 reference stations, the YNDO, JEOJ, PALM, and MARA reference stations were used as the 4 reference stations, and the JEOJ, PALM, and MARA reference stations were used as the 3 reference stations. In the case of the 2 reference stations, the JEOJ and MARA reference stations, which have the longest baseline distance, were used. The pseudorange correction provided from the reference station was collected and regenerated as the pseudorange correction of rover station location using the pseudorange correction regeneration algorithm which utilizes the exponential model developed in this paper. The result was compared by applying the regenerated pseudorange correction to positioning, which is presented in Figs. 3 and 4 and Tables 5 and 6. Fig. 3 shows the horizontal error of the positioning which applies the regenerated pseudorange correction of MUJU rover station obtained by changing the number of reference stations. $\Delta \mathrm{N}$ of $\mathrm{Y}$-axis represents the northsouth direction error, and $\Delta \mathrm{E}$ of $\mathrm{X}$-axis represents the eastwest direction error. The central location $(0,0)$ represents the notified coordinate of MUJU rover station. Fig. $3 a$ is when the pseudorange correction was regenerated using 2 reference stations, and bias is observed in a southward direction. Fig. $3 \mathrm{~b}$ is when 3 reference stations are used, and bias is observed in an eastward direction. Figs. 3c and $3 d$ are when 4 and 5 reference stations are used, and it is found that bias is reduced and accuracy is improved compared to when 2 and 3 reference stations are used. Fig. 4 shows the vertical error depending on time. $\mathrm{X}$-axis represents the time and $\Delta \mathrm{V}$ of $\mathrm{Y}$-axis represents the vertical error. Fig. 


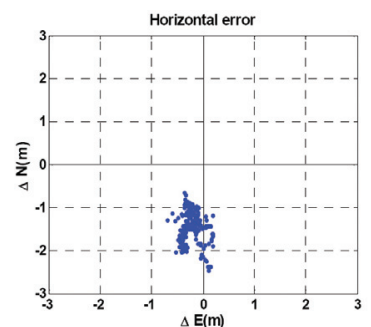

(a) two reference stations

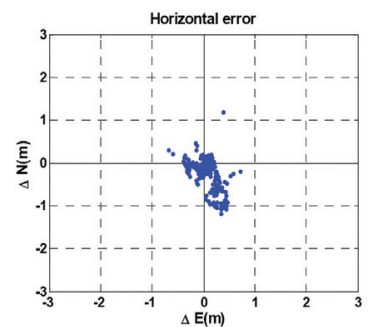

(c) four reference stations

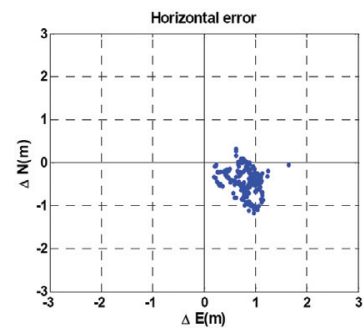

(b) three reference stations

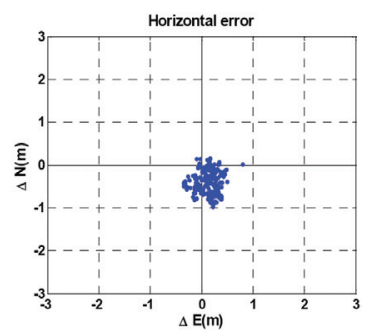

(d) five reference stations

Fig. 3. Horizontal error.

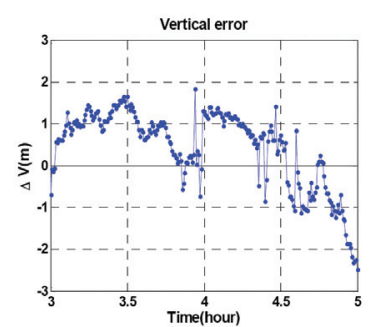

(a) two reference stations

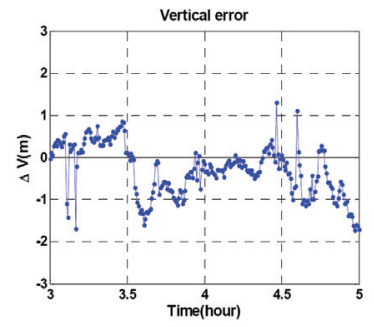

(c) four reference stations
Fig. 4. Vertical error.

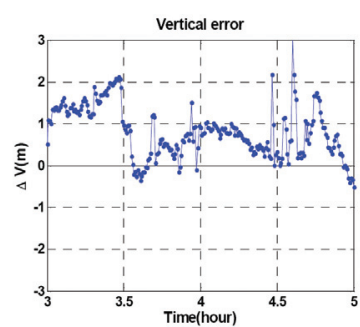

(b) three reference stations

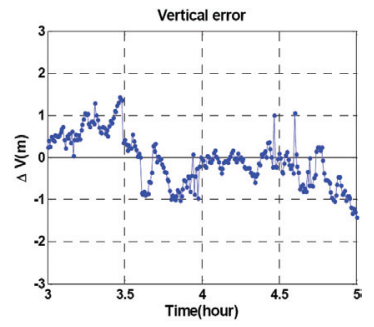

(d) five reference stations
$4 \mathrm{a}$ is when 2 reference stations are used, Fig. $4 \mathrm{~b}$ is when 3 reference stations are used, Fig. $4 \mathrm{c}$ is when 4 reference stations are used, and Fig. $4 \mathrm{~d}$ is when 5 reference stations are used. When 4 reference stations are used, anomalous values are observed at around 03:10. This is due to the effect of noise that occurred in the pseudorange correction of YNDO reference station. In Figs. $4 \mathrm{a}$ and $4 \mathrm{~b}$ where the YNDO reference station is not included, the anomalous values are not observed at the corresponding time. When 5 reference stations are used, the effect of noise that occurred in the pseudorange correction of YNDO reference station becomes smaller as the baseline of YNDO reference station is longer
Table 5. RMSE result (meter).

\begin{tabular}{lllll}
\hline & $\begin{array}{l}\text { Two } \\
\text { Reference } \\
\text { stations }\end{array}$ & $\begin{array}{l}\text { Three } \\
\text { Reference } \\
\text { stations }\end{array}$ & $\begin{array}{l}\text { Four } \\
\text { Reference } \\
\text { stations }\end{array}$ & $\begin{array}{l}\text { Five } \\
\text { Reference } \\
\text { stations }\end{array}$ \\
\hline Horizontal Error & 1.49 & 1.37 & 0.50 & 0.50 \\
Vertical Error & 1.02 & 0.87 & 0.68 & 0.60 \\
\hline
\end{tabular}

Table 6. Bias result (meter).

\begin{tabular}{lllll}
\hline & $\begin{array}{l}\text { Two } \\
\text { Reference } \\
\text { stations }\end{array}$ & $\begin{array}{l}\text { Three } \\
\text { Reference } \\
\text { stations }\end{array}$ & $\begin{array}{l}\text { Four } \\
\text { Reference } \\
\text { stations }\end{array}$ & $\begin{array}{l}\text { Five } \\
\text { Reference } \\
\text { stations }\end{array}$ \\
\hline North & -1.42 & -0.52 & -0.25 & -0.38 \\
East & -0.22 & 0.92 & 0.07 & 0.11 \\
Vertical & 0.44 & 0.46 & -0.30 & -0.03 \\
\hline
\end{tabular}

Table 7. SRD \& IDW \& EXP RMSE result (meter).

\begin{tabular}{lccc}
\hline & SRD & IDW & EXP \\
\hline Horizontal Error & 0.73 & 0.56 & 0.50 \\
Vertical Error & 1.14 & 0.86 & 0.68 \\
\hline
\end{tabular}

Table 8. SRD \& IDW \& EXP Bias result (meter).

\begin{tabular}{lccc}
\hline & SRD & IDW & EXP \\
\hline North & 0.53 & -0.13 & -0.25 \\
East & -0.11 & 0.39 & 0.07 \\
Vertical & -0.34 & 0.65 & -0.30 \\
\hline
\end{tabular}

than that of CCHJ reference station. Thus, relatively small noise is observed compared to when 4 reference stations are used.

Table 5 shows the positioning RMS error of MUJU rover station depending on the number of reference stations. When the pseudorange correction was regenerated using 4 and 5 reference stations, the accuracy was improved by $0.99 \mathrm{~m}$ in the horizontal direction compared to when 2 reference stations are used, and by $0.87 \mathrm{~m}$ in the horizontal direction compared to when 3 reference stations are used. If it is compared between when 4 and 5 reference stations are used, the error was the same in the horizontal direction. When 5 reference stations are used, the error was reduced by $0.08 \mathrm{~m}$ in the vertical direction compared to when 4 reference stations are used. Table 6 shows the averages of bias for the North, East, and vertical directions depending on the number of reference stations. When 4 and 5 reference stations are used, the bias was less than $0.50 \mathrm{~m}$ in every direction. Through this, it is found that the error and bias decrease abruptly when 4 or more reference stations are used.

\subsection{Comparison of algorithm accuracy}

To test the algorithm developed in this paper, it was 
compared with the positioning result of DGPS based on single reference station and the result of inverse distance weighting developed in the previous study. Fig. 5 shows the horizontal error, and Fig. 6 shows the vertical error. Table 7 shows the result of RMS error, and Table 8 shows the averages of bias. For the DGPS positioning based on single reference station, the pseudorange correction of $\mathrm{CCHJ}$ reference station was used, and the $\mathrm{CCHJ}$ reference station is located relatively close to the MUJU rover station among the reference stations operated by the DGNSS Central Office. The pseudorange correction was regenerated using 4 reference stations because the accuracy of less than $1 \mathrm{~m}$ could be obtained when the pseudorange correction is regenerated using 4 or more reference stations as demonstrated by the comparison of positioning accuracy depending on the number of reference stations. In this paper, the algorithm developed for the pseudorange correction regeneration is expressed as EXP, the inverse distance weighting is expressed as IDW, and the DGPS based on single reference station is expressed as SRD.

In Table 7, EXP shows a smaller error than SRD and IDW when the horizontal and vertical errors for each method are compared. In the case of IDW, the bias for the North direction was reduced compared to SRD, but the biases for the East and vertical directions were increased. As for EXP, the bias was reduced in every direction compared to SRD as shown in Table 8. Based on this, it is demonstrated that the accuracy of EXP is higher than that of SRD and IDW.

\section{CONCLUSION}

In this paper, a pseudorange correction regeneration algorithm was developed for improving the positioning accuracy of DGPS based on multi-reference stations in the Korean Peninsula, and the optimal minimum number of reference stations necessary for the DGPS based on multireference stations was determined by changing the number of reference stations when regenerating the pseudorange correction. The research was conducted using 2 5 reference stations, and an error of less than $1 \mathrm{~m}$ could be obtained when the pseudorange correction is regenerated using 4 or more reference stations. Therefore, it is found that a certain level of positioning accuracy can be achieved for the DGPS using multiple reference stations when the pseudorange corrections are collected from at least 4 reference stations and regenerated as the pseudorange correction of rover station location. After determining the optimal minimum

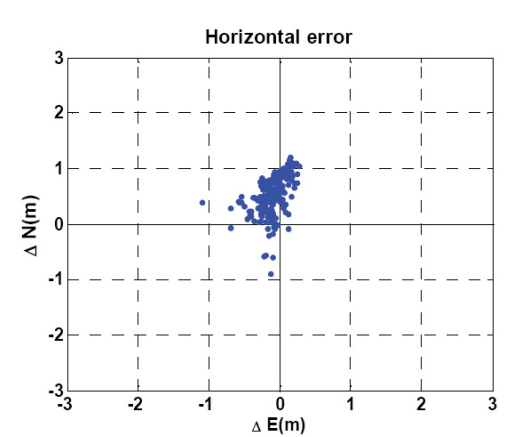

(a) SRD

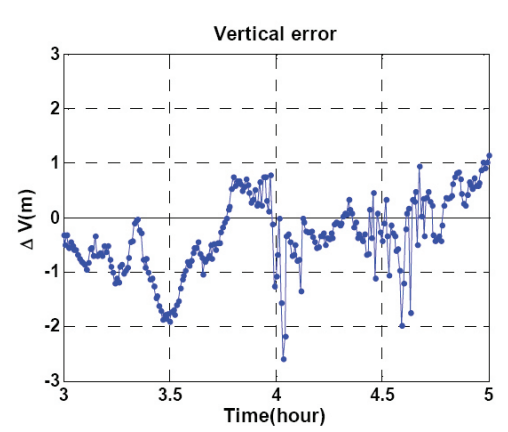

(a) SRD

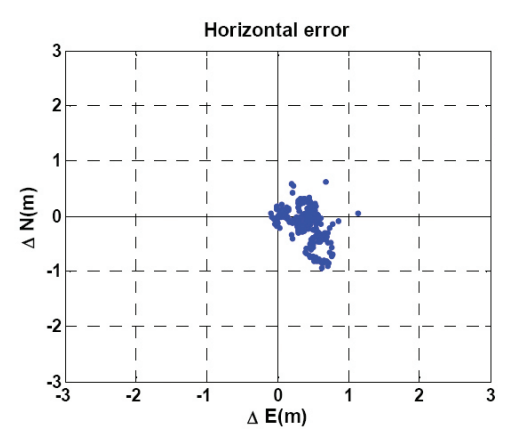

(b) IDW

Fig. 5. SRD \& IDW \& EXP Horizontal error.

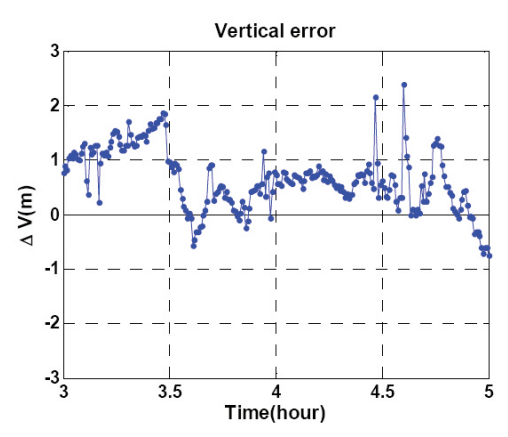

(b) IDW

Fig. 6. SRD \& IDW \& EXP Vertical error.

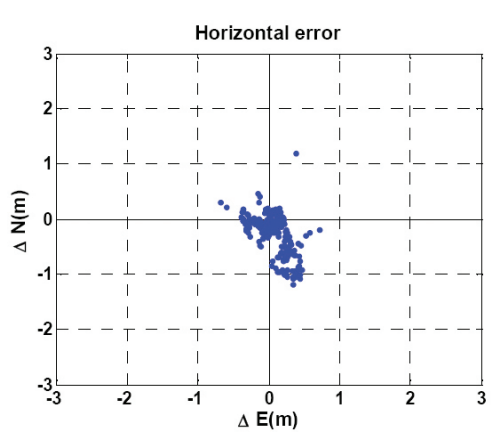

(c) EXP

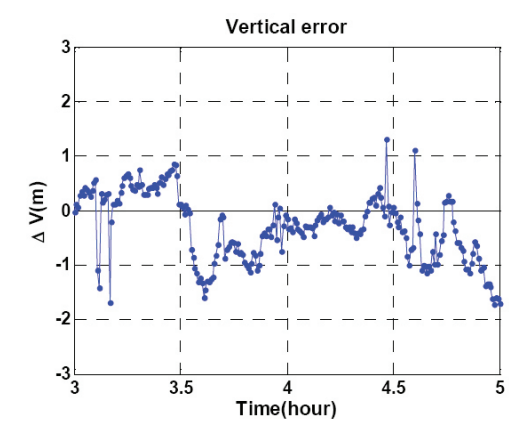

(c) EXP 
number of reference stations necessary for the DGPS based on multi-reference stations, the pseudorange correction regeneration algorithm proposed in this paper was tested by comparing with the result of previous study. The pseudorange correction regeneration algorithm proposed in this paper was developed using an exponential model, and the weight on baseline distance was applied differently by assigning weight depending on the difference of the longest and shortest baselines among the baseline distances between reference stations and rover station. To evaluate the accuracy of algorithm, it was compared with the result of inverse distance weighting developed in the previous study. The positioning accuracy of the algorithm developed in this paper was found to be improved by $19 \%$ compared to the inverse distance weighting.

The pseudorange correction regeneration algorithm developed in this paper is optimized to be used in a small country such as the Korean Peninsula rather than a range of several thousand kilometers. The algorithm was developed so that high accuracy is achieved by changing the weight coefficient depending on the baseline distance difference of reference station and rover station. Thus, for applying the algorithm to the research related with DGPS and the practical life within a range of $500 \mathrm{~km}$, evaluation needs to be performed over a long period in the future for the entire Korean Peninsula.

\section{ACKNOWLEDGMENTS}

This work was supported by "Development of GNSSbased Transportation Technology" which is funded by the Ministry of Land, Transportation and Maritime Affairs, Korea.

\section{REFERENCES}

Abousalem, M. A. 1997, Performance Overview of Two WADGPS Algorithms, GPS World, 8, 48-58

Bae, J. S., Kim, S. Y., \& Kee, C. D. 2003, Application and Implementation of IDGPS using Virtual Reference Station, Journal the Korean Society for Aeronautical \& Space Sciences, 15, 432-435

Kim, H. I. \& Park, K. D. 2011, A Study on the PRC Generation Algorithms for Virtual Reference Stations Using a Network of DGNSS Reference Stations, Journal of the Korean Society of Survey, Geodesy, Photogrammetry, and Cartography, 29, 221-228

Kim, I. S. 2010, Availability Evaluation of Network DGPS
Positioning for Various Facilities Management in Dense Housing Area, Journal of the Korean Society for GeoSpatial Information System, 18, 93-99

Koo, J., Pyo, S. H., Kang, K. S., \& Kim, K. H. 2011, Analysis of DGPS Approach and Landing Accuracy using Air Base Precision approach Radar, Journal of the Korea Institute of Military Science and Technology, 14, 788-797

Lee, S. J., Chang, J. W., Jeon, B. H., Seong, K. J., \& Yeom, C. H. 2009, Comparison Study on Take-Off and Landing Fight Test Using Ground Observation and DGPS Method, Journal the Korean Society for Aeronautical \& Space Sciences, 37, 931-938

Loomis, T., Sheynblatt, L., \& Mueller, T. 1991, Differential GPS Network Design, in 1991 ION, Albuquerque, 11-13 Sep 1991

Mueller, T. 1994, Minimum Variance Network DGPS Algorithm, in 1994 TAU Corporation, Las Vegas, NV, 1115 Apr 1994

Oh, J. T. \& Lee, S. Y. 2010, Study of Risky Driving Decision Device using DGPS/RTK, Journal of the Korean Society of Civil Engineers, 30, 303-311

Song, B. S., Oh, K. R., \& Kim, J. H. 2006, Analysis of PRC Regeneration Algorithm Performance in Dynamic Environment by using Multi-DPGS Signal, Journal of the Korea Information Processing Society, 13A, 335-342

Tang, W., Johnson, N., \& Graff, J. 1989, Differential GPS Operation with Multiple Ground Reference Stations, in 1989 ION, Colorado Springs, Colorado, 27-29 Sep 1989

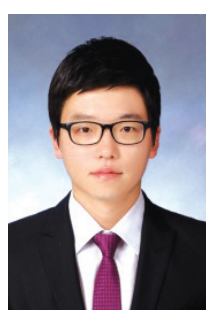

Koon-Tack Kim received his B.S. and M.S. degrees in the Geoinformatic Engineering from Inha University in 2010 and 2013, respectively. He has joined the Satellite Navigation Team at KARI since 2013 and His current research is the development of GNSS-based transportation technology.

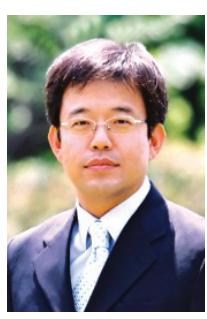

Kwan-Dong Park received his B.S. in Mechanical Engineering from Hanyang University in 1990, and M.S. and Ph.D. in Aerospace Engineering from the University of Texas at Austin in 1992 and 2000, respectively. From 2001 through 2002, he worked as a post-doc fellow at HarvardSmithsonian Center for Astrophysics. Since 2007, he is an associate professor in the Department of Geoinformatic Engineering at Inha University. His research interests include precise positioning algorithm development based on GNSS measurements, and satellite applications. 


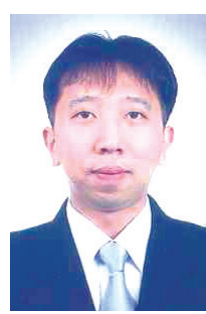

Eunseong Lee is a senior researcher at Korea Aerospace Research Institute. He was a research staff member in the Mechanical and Aerospace Engineering at the University of California, Los Angeles. He received his Ph.D. in Aerospace Engineering from Konkuk University in 2005. He received his M.S. degree in Aerospace Engineering from the Konkuk University, Korea in 1998. His research areas include GPS RTK, orbit determination of satellites, and integrity of GPS augmentation systems.

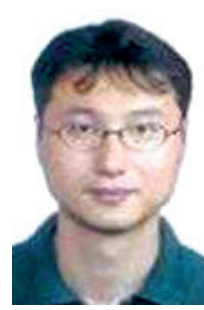

Moon Beom Heo received a B.S. in the Mechanical Engineering from Kyung-Hee University, Korea, in 1992, and the M.S. and Ph.D. degrees in the Mechanical and Aerospace Engineering from the Illinois Institute of Technology, Chicago, respectively, in 1997 and 2004. He is currently a head of the Satellite Navigation Team at the Korea Aerospace Research Institute, Korea. His work is focused on Global Navigation Satellite System and their augmentation. 